the $\mathrm{N}$ terminus is also important to limit proteolysis. After proteasomal digestion, MHC class I peptides often have a definitive $\mathrm{C}$ terminal residue but not a final $\mathrm{N}$ terminus (10). The latter will be produced by aminopeptidases present in the cytoplasm and/or the ER, such as ERAP (11). The second important function of the termini of a peptide is for its translocation from the cytoplasm to the ER and preferential binding to the TAP transporter. Indeed, the three $\mathrm{N}$ terminal positions and the $\mathrm{C}$ terminal residue of cytoplasmic peptides are critical in the selection of substrates by TAP and their efficient transport (12). The final role of the termini of a peptide is in the process of its binding to MHC class I molecules. At 8,9 , and even 10 residues in length, peptide binding to MHC class I can be optimal and satisfy anchoring to pockets (13). Beyond this length, MHC does not exclude binding altogether, but binding becomes suboptimal by allowing only the extremities of the peptide to be associated with the MHC molecule. The peptide-MHC class I complexes that are produced by 12-, 13-, or even 14mer peptides (14) are structurally unique with a central bulge that will be recognized and flattened by the $T$ cell receptor upon binding (15). Thus, the flanking residues of an MHC class I epitope can influence processing, transport, MHC binding, and T cell recognition. These considerations are critical for the design of peptide-based vaccines, but their prediction is often difficult and requires in vivo models to be confirmed.

It is clear from the studies by Wahlström et al. (3) and Le Gall et al. (7) that appear in this issue of the JCI that the lessons we can learn from antigen presentation are some of the most challenging to translate into therapeutic approaches, but nobody should be afraid of a challenge that might bring huge returns.

\section{Acknowledgments}

L. Teyton is supported by the NIH.

Address correspondence to: Luc Teyton, Department of Immunology, The Scripps Research Institute, 10550 North Torrey Pines Road, La Jolla, California 92037, USA. Phone: (858) 784-2728; Fax: (858) 784-8805; E-mail: 1teyton@scripps.edu.

1. Bjorkman, P.J., et al. 1987. Structure of the human class I histocompatibility antigen, HLA-A2. Nature. 329:506-512.

2. Townsend, A.R., et al. 1986. The epitopes of influenza nucleoprotein recognized by cytotoxic $\mathrm{T}$ lymphocytes can be defined with short synthetic peptides. Cell. 44:959-968.

3. Wahlström, J., et al. 2007. Identification of HLA-DRbound peptides presented by human bronchoalveolar lavage cells in sarcoidosis. J. Clin. Invest. 117:3576-3582. doi:10.1172/JCI32401.

4. Berlin, M., Fogdell-Hahn, A., Olerup, O., Eklund, A., and Grunewald, J. 1997. HLA-DR predicts the prognosis in Scandinavian patients with pulmonary sarcoidosis. Am. J. Respir. Crit. Care Med. 156:1601-1605.

5. Katchar, K., Wahlstrom, J., Eklund, A., and
Grunewald, J. 2001. Highly activated T-cell receptor AV2S3(+) CD4(+) lung T-cell expansions in pulmonary sarcoidosis. Am.J. Respir. Crit. Care Med. 163:1540-1545.

6. Hunt, D.F., et al. 1992. Characterization of peptides bound to the class I MHC molecule HLA-A2.1 by mass spectrometry. Science. 255:1261-1263.

7. Le Gall, S., Stamegna, P., and Walker, B.D. 2007. Portable flanking sequences modulate CTL epitope processing. J. Clin. Invest. 117:3563-3575. doi:10.1172/JCI32047.

8. Takahashi, H., et al. 1989. Structural requirements for class I MHC molecule-mediated antigen presentation and cytotoxic $\mathrm{T}$ cell recognition of an immunodominant determinant of the human immunodeficiency virus envelope protein. J. Exp. Med. 170:2023-2035.

9. Kaul, R., et al. 2001. CD8 ${ }^{+}$lymphocytes respond to different HIV epitopes in seronegative and infected subjects. J. Clin. Invest. 107:1303-1310.

10. Cascio, P., Hilton, C., Kisselev, A.F., Rock, K.L., and Goldberg, A.L. 2001. 26S proteasomes and immunoproteasomes produce mainly $\mathrm{N}$-extended versions of an antigenic peptide. EMBO J. 20:2357-2366

11. Yan, J., et al. 2006. In vivo role of ER-associated peptidase activity in tailoring peptides for presentation by MHC class Ia and class Ib molecules. J. Exp. Med. 203:647-659.

12. Uebel, S., et al. 1995. Requirements for peptide binding to the human transporter associated with antigen processing revealed by peptide scans and complex peptide libraries. J. Biol. Chem. 270:18512-18516.

13. Fremont, D.H., Matsumura, M., Stura, E.A., Peterson, P.A., and Wilson, I.A. 1992. Crystal structures of two viral peptides in complex with murine MHC class I H-2Kb. Science. 257:919-927.

14. Probst-Kepper, M., et al. 2004. Conformational restraints and flexibility of 14 -meric peptides in complex with HLA-B*3501. J. Immunol. 173:5610-5616.

15. Tynan, F.E., et al. 2007. A T cell receptor flattens a bulged antigenic peptide presented by a major histocompatibility complex class I molecule. Nat. Immunol. 8:268-276.

\title{
A nervous breakdown in the skin: stress and the epidermal barrier
}

\author{
Andrzej Slominski
}

Department of Pathology and Laboratory Medicine, Health Science Center, University of Tennessee, Memphis, Tennessee, USA.

\begin{abstract}
In this issue of the JCI, Aberg and colleagues report that psychological stress disrupts the skin's antimicrobial barrier and increases the severity of cutaneous infections (see the related article beginning on page 3339). This effect is mediated by endogenous glucocorticoids produced secondarily to stressrelated activation of the hypothalamic-pituitary-adrenal axis. Thus, this study provides what I believe to be the first mechanistic link between psychological stress and increased susceptibility to microbial infection.
\end{abstract}

Nonstandard abbreviations used: CRF, corticotropin-releasing factor; CRF1, CRF receptor type 1; HPA, hypothalamic-pituitary-adrenal; MSH, melanocytestimulating hormone; POMC, proopiomelanocortin.

Conflict of interest: The author has declared that no conflict of interest exists.

Citation for this article: J. Clin. Invest. 117:3166-3169 (2007). doi:10.1172/JCI33508.
Skin changes during systemic stress The main function of the epidermis is to regulate epidermal permeability and to act as a physical, chemical, and antimicrobial defense system via the actions of the outermost layer of the epidermis, the stratum corneum (1). The natural antimicrobial defenses of the skin also involve elements of the innate immune response such as the production of antimicrobial peptides, lipids, Toll-like receptors, proinflammatory cytokines, and chemokines (1). During periods of psychological stress the cutaneous homeostatic permeability barrier is disturbed, as is the integrity and protective function of the stratum corneum (1). Furthermore, a large number of skin diseases, including atopic dermatitis and psoriasis, appear to be precipitated or exacerbated by psychological stress (2). Nevertheless, the underlying mechanism of a specific pathogenic role for psychological stress in skin pathology has remained unclear. 


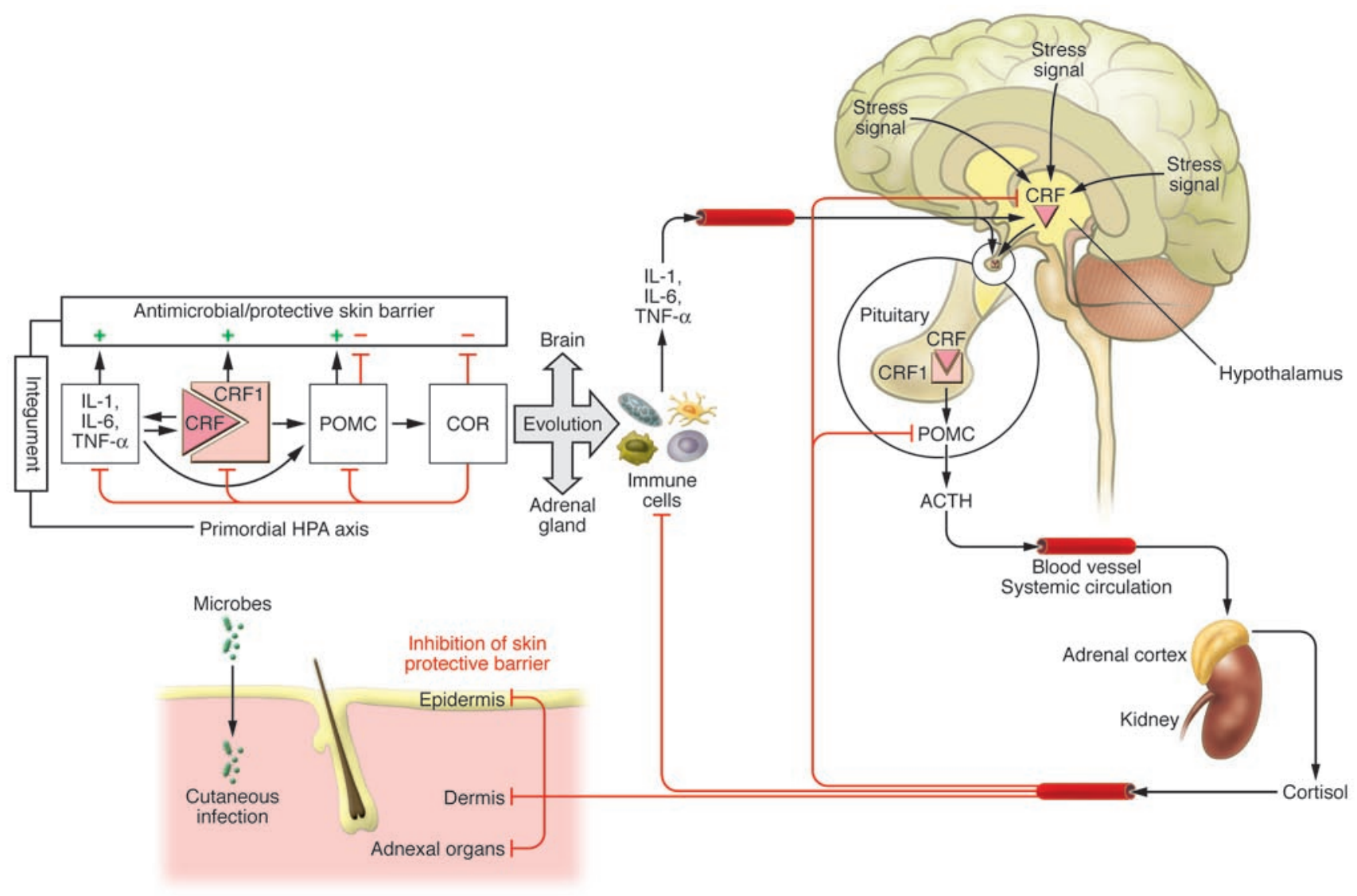

Figure 1

Evolution of the HPA axis. The primordial HPA axis (left) may have developed first in the integument for the regulation of its defensive activity against the hostile environment and pathogens. At its center are the CRF-related peptide(s) that through CRF1 (an integrating receptor) coordinate innate immune activity and protective barrier formation directly or indirectly via the expression of the proinflammatory cytokines IL-1, IL-6, and TNF- $\alpha$. An inhibitory feedback loop begins with CRF1-activated POMC-derived ACTH production/secretion and culminates with production/secretion of cortisol or corticosterone (COR) that "shuts off" HPA axis activity and inhibits the antimicrobial and protective functions of the skin barrier. The intermediate signaling molecules (POMC peptides) can both weaken the skin protective barrier via their immunosuppressive action, and strengthen it through stimulation of melanogenesis or direct antimicrobial effects. Thus, the antimicrobial and protective barrier functions were regulated and fine-tuned by the primordial HPA, because of the close association of all of its elements. During evolution, the main signaling elements of the primordial HPA axis (CRF $\rightarrow$ CRF $1 \rightarrow$ POMC $\rightarrow$ ACTH $\rightarrow$ cortisol or corticosterone) may have been adapted and perfected by the central neuroendocrine system to form the HPA axis (right panel), which has separated in space and overall function from the immune system and skin. The immune system retains its stimulatory activity via cytokines. However, as Aberg et al. show in this issue of the $\mathrm{JCl}$, the skin antimicrobial protective barrier is weakened by psychological stress-induced production of systemic corticosteroids (7), an unintended side effect. In this context, the cutaneous HPA axis has only low local activity and serves as an evolutionary record of the parental system.

\section{Hypothalamic-pituitary-adrenal axis}

The main adaptive response to sustained systemic stress is mediated by the hypothalamic-pituitary-adrenal (HPA) axis (Figure 1). This response begins with the stress-induced hypothalamic production of corticotropin-releasing factor (CRF), which, in the anterior pituitary, activates CRF receptor type 1 (CRF1) and induces the production and release of the proopiomelanocortin-derived (POMC-derived) adrenocorticotropic hormone (ACTH) (3-5). Psychological stress can also activate the HPA axis and many of the adverse effects of psychological stress on the body's functions are secondary to sustained HPA activity $(3,4)$. The expression of CRF and POMC can also be stimulated by proinflammatory cytokines (6), thus involving the immune system in the central regulation of the HPA axis. Pituitaryderived ACTH stimulates adrenocortical production and secretion of glucocorticoids such as the steroid hormone cortisol (in humans) and corticosterone (in rodents) (4). These glucocorticoids counteract the effects of stressors, suppress the immune system, and attenuate the functional activity of the HPA axis via feedback inhibition of CRF and POMC expression.

\section{Psychological stress disrupts the natural antimicrobial defenses of the epidermis}

In this context, the study reported by Aberg and colleagues in this issue of the JCI (7) represents a timely contribution to our understanding of the deleterious effect of psychological stress on epidermal function. These authors demonstrate psychological stressinduced alterations in the structure and function of the stratum corneum, causing the downregulation of epidermal and adnexal expression of antimicrobial peptides and an increased severity of cutaneous infection in mice. These effects were accompanied by 
increased glucocorticoid production, could be mimicked by systemic glucocorticoid administration, and attenuated by administration of the CRF1 antagonist antalarmin and also by administration of the glucocorticoid receptor antagonist RU-486. Furthermore, antalarmin, RU-486, and adrenalectomy were shown to enhance the otherwise low constitutive expression of antimicrobial peptides in these animals. Because homeostasis of the permeability barrier was restored by the use of CRF1 or glucocorticoid receptor antagonists, the authors concluded that psychological stress inhibits the antimicrobial action of the epidermal and adnexal compartments via glucocorticoid-induced HPA axis activation. They also propose what they believe to be a novel therapeutic approach to the treatment of psychological stress disruption of the cutaneous and extracutaneous epithelial barrier defense mechanisms: pharmacological blockade of CRF1 or glucocorticoid receptors in order to attenuate the activity of the regulatory and/or functional arms of the HPA axis.

The major strength of the current work is represented by its conceptual and clinical implications (7). However, data on the functional parameters of the HPA axis are limited, since neither plasma nor skin corticosterone, POMC, ACTH, or CRF levels were measured or correlated with circadian rhythm (under physiological conditions, plasma ACTH and glucocorticoid levels fluctuate in a circadian fashion). Future studies, including experiments in genetically modified mice in which the genes encoding CRF1, POMC, or glucocorticoidregulating enzymes and their receptors are altered, will hopefully provide proof of concept of this proposed mechanism. It must also be acknowledged that the use of animal models (rodents) to test the proposed connection between protective barrier function, psychological stress, and HPA axis activity is limited because of the fundamental differences in skin physiology and pathology between rodents and humans. For example, mice are nocturnal species, while human skin is continuously exposed to solar radiation during the daytime (8). These differences are strikingly associated with specific anatomic and histologic characteristics of epidermal and adnexal compartments and their biological activities. For instance, dramatic changes in skin morphology and physiologic function have been previously shown to synchronize with alterations in the hair follicle cycle in mice, an effect not seen in human skin $(8,9)$. Thus, the dynam- ic interactions between the brain and skin (by way of psychological stress) should optimally be confirmed in humans.

\section{The cutaneous HPA system}

Throughout evolution, the skin - being continuously exposed to hostile biological, chemical, or physical (i.e., solar or thermal energy) factors - had to develop efficient sensory and signaling capabilities to differentially react to changes in the external environment in order to protect, restore, or maintain the local and global homeostasis that is critical for survival. We have previously proposed that the precise coordination and execution of local homeostatic responses are mediated by a cutaneous neuroendocrine system, able to reset adaptation mechanisms through rapid (neural) or slow (humoral) pathways acting at the local or systemic level (8). The impact of psychological stress must be considered in the context of this bidirectional pathway of communication between the brain and skin (10).

Human skin indeed expresses elements of the HPA axis including CRF, CRF1, and POMC as well as the processing machinery that generates ACTH, $\beta$-endorphin, and melanocyte-stimulating hormone (MSH) peptides (in a regulated and spatiotemporally restricted manner) and their corresponding receptors $(11,12)$. Skin cells also express the enzymatic machinery involved in the sequential metabolism of cholesterol to the steroid hormone pregnenolone and to corticosteroids, and produce endogenous cortisol and corticosterone $(8,13)$, albeit at very low levels (14). These elements may be organized in functional, cell type-specific regulatory loops within the structural hierarchy of the $\mathrm{HPA}$ axis, (e.g., $\mathrm{CRF} \rightarrow \mathrm{CRF} 1 \rightarrow \mathrm{POMC} \rightarrow$ $\mathrm{ACTH} \rightarrow$ cortisol and/or corticosterone), in which CRF-induced production of corticosteroids is dependent on both CRF1 activity and expression of POMC $(8,12,13)$. Environmental stressors such as ultraviolet radiation stimulate expression of CRF, POMC, and their corresponding receptors $(11,12,15,16)$, while negative feedback regulation of cutaneous CRF expression by cortisol has also been demonstrated (17). Thus, differential, CRF1driven responses in the skin reproduce key features of the central HPA axis at both the whole-tissue and single-cell levels $(12,13)$.

\section{Merging of the central and peripheral stress response systems}

Because the HPA axis coordinates an adaptive response to counteract the actions of stressors, stabilize organ function, and restore general homeostasis, one is faced with an intellectual dilemma. How can a system designed to protect whole-body homeostasis (via the HPA axis) endanger the organism's survival through disruption of epidermal barrier function on multiple levels? I propose that during evolution the fundamental mechanism of the stress response, the HPA axis, developed first in the integument to promote species survival and was then adapted and perfected by the CNS and endocrine system (Figure 1). This primordial HPA in the integument was composed of CRF, POMC, and steroid signaling pathways that act in concert with the innate immune system in order to create the optimal response against pathogens and other stressors and protect internal homeostasis. In this system, CRF, acting directly through CRF1, stimulates skin immune responses through the stimulation of NF- $\mathrm{KB}$ to increase production of IL-1, IL-6, and TNF- $\alpha$ (12). This system can also be activated by pathogens in the form of lipopolysaccharides and bacterial antigens. CRF production is induced, which in turn stimulates proinflammatory cytokines through activation of CRF1 (18). This self-amplifying innate immune response is then interrupted by an inhibitory loop mediated by CRF1-activated production of POMC peptides (ACTH, $\alpha$-MSH, and $\beta$-endorphin, which have antiinflammatory properties; ref. 19) and finally via corticosteroids induced by ACTH (Figure 1). In the context of epidermal barrier formation, it is significant that activation of CRF1 stimulates keratinocyte survival (perhaps through activation of NF- $\mathrm{KB}$ ) and increases keratinocyte differentiation (through $\mathrm{Ca}^{2+}$ signaling) (12), and POMC-derived melanocortins stimulate production of protective melanin pigment (15), increase melanocyte survival, and decrease ultraviolet radiationinduced DNA damage (20). The latter integumental protective properties would counterbalance the potent immunoinhibitory action of $\alpha$-MSH $(11,19)$. Throughout evolution, this autoregulatory circuitry founded by the primordial integumental HPA axis could have undergone specialization and separation of its functional components in space and time (Figure 1). In becoming detached from its point of origin the HPA axis lost its original purpose (i.e., forming of a protective barrier), while retaining its cutaneous inhibitory activities through the action of cortisol or corticosterone.

The central biological paradigm - that regulatory mechanisms, which provide significant selective advantages, are reused in multiple tissues throughout evolution - is 
clearly illustrated by the redundancy in organizational structure of the stress response systems in the CNS and the skin $(8,13)$. This redundancy has its functional consequences when psychological stress-mediated activation of central HPA axis signaling negatively affects protective and antimicrobial skin barrier function, as is demonstrated in this issue by Aberg et al. (7). These findings will hopefully have clinical impact by stimulating the development of systemic and topical selective receptor antagonists for peptide and steroidal messengers of the HPA axis, as well as topical use of stimulators of cortisol metabolic inactivation, and the use of inhibitors of steroidogenesis in order to improve the antimicrobial and protective barrier activity of the skin. This may also be the dawn of a new clinical approach for the treatment of psychological stress-induced inflammatory dermatoses via the use of systemic or topical CRF1 antagonists.

\section{Acknowledgments}

Comments from Jacobo Wortsman and Curtis Dohan as well as NIH support (grant AR052190) are acknowledged.

Address correspondence to: Andrzej Slominski, Department of Pathology and
Laboratory Medicine, Health Science Center, University of Tennessee, 930 Madison Avenue, Memphis, Tennessee 38163, USA. Phone: (901) 448-3741; Fax: (901) 4486979; E-mail: aslominski@utmem.edu.

1. Elias, P.M. 2005. Stratum corneum defensive functions: an integrated view. J. Invest. Dermatol. 125:183-200.

2. Arck, P.C., Slominski, A., Theoharides, T.C., Peters, E.M., and Paus, R. 2006. Neuroimmunology of stress: skin takes center stage. J. Invest. Dermatol. 126:1697-1704.

3. McEwen, B.S. 2007. Physiology and neurobiology of stress and adaptation: central role of the brain. Physiol. Rev. 87:873-904.

4. Chrousos, G.P. 1995. The hypothalamic-pituitaryadrenal axis and immune-mediated inflammation. N. Engl. J. Med. 332:1351-1362.

5. Vale, W., Spiess, J., Rivier, C., and Rivier, J. 1981. Characterization of a 41-residue ovine hypothalamic peptide that stimulates secretion of corticotropin and beta-endorphin. Science. 213:1394-1397.

6. Turnbull, A.V., and Rivier, C. 1995. Regulation of the HPA axis by cytokines. Brain Behav. Immun. 9:253-275.

7. Aberg, K.M., et al. 2007. Psychological stress downregulates epidermal antimicrobial peptide expression and increases severity of cutaneous infections in mice. J. Clin. Invest. 117:3339-3349. doi:10.1172/ JCI31726.

8. Slominski, A., and Wortsman, J. 2000. Neuroendocrinology of the skin. Endocr. Rev. 21:457-487.

9. Stenn, K.S., and Paus, R. 2001. Controls of hair follicle cycling. Physiol. Rev. 81:449-494.

10. Paus, R., Theoharides, T.C., and Arck, P.C. 2006 Neuroimmunoendocrine circuitry of the 'brain- skin connection'. Trends Immunol. 27:32-39.

11. Slominski, A., Wortsman, J., Luger, T., Paus, R., and Solomon, S. 2000. Corticotropin releasing hormone and proopiomelanocortin involvement in the cutaneous response to stress. Physiol. Rev. 80:979-1020.

12. Slominski, A., et al. 2006. Corticotropin releasing hormone and the skin. Front. Biosci. 11:2230-2248.

13. Slominski, A., Wortsman, J., Tuckey, R.C., and Paus, R. 2007. Differential expression of HPA axis homolog in the skin. Mol. Cell. Endocrinol. 265-266:143-149.

14. Slominski, A., et al. 2004. A novel pathway for sequential transformation of 7-dehydrocholesterol and expression of the P450scc system in mammalian skin. Eur. J. Biochem. 271:4178-4188.

15. Slominski, A., Tobin, D.J., Shibahara, S., and Wortsman, J. 2004. Melanin pigmentation in mammalian skin and its hormonal regulation. Physiol. Rev. 84:1155-1228.

16. Zbytek, B., Wortsman, J., and Slominski, A. 2006. Characterization of a ultraviolet B-induced corticotropin-releasing hormone-proopiomelanocortin system in human melanocytes. Mol. Endocrinol. 20:2539-2547.

17. Ito, N., et al. 2005. Human hair follicles display a functional equivalent of the hypothalamic-pituitary-adrenal axis and synthesize cortisol. FASEB J. 19:1332-1334.

18. Zbytek, B., and Slominski, A.T. 2007. CRH mediates inflammation induced by lipopolysaccharide in human adult epidermal keratinocytes. J. Invest. Dermatol. 127:730-732.

19. Luger, T., Paus, R., Lipton, J., and Slominski, A. 1999. Cutaneous neuromodulation: the proopiomelanocortin System. Ann. N. Y. Acad. Sci. 885:1-479.

20. Bohm, M., Luger, T.A., Tobin, D.J., and Garcia-Borron, J.C. 2006. Melanocortin receptor ligands: new horizons for skin biology and clinical dermatology. J. Invest. Dermatol. 126:1966-1975.

\title{
OX40 signaling directly triggers the antitumor effects of NKT cells
}

\author{
Dapeng Zhou
}

Department of Melanoma Medical Oncology, Division of Cancer Medicine, University of Texas MD Anderson Cancer Center, Houston, Texas, USA.

\begin{abstract}
Pathways involving the costimulatory molecule OX40 and OX40 ligand $(\mathrm{OX} 40 \mathrm{~L})$ enhance tumor rejection. It was presumed that this effect was mediated by changes in DCs and/or T cells. In this issue of the JCI, Zaini et al. report that, in mice, intratumoral injection of DCs genetically modified to express OX40L suppressed the growth of a preexisting melanoma by directly triggering an antitumor NKT cell response (see the related article beginning on page 3330). This work suggests that the intratumoral NKT cell population may be harnessed for cancer immunotherapy and that $\mathrm{OX} 40$ costimulation may be used as a unique trigger of the antitumor activity of these cells.
\end{abstract}

The effective $\mathrm{T}$ cell response to a tumor antigen requires not only stimulation by

Nonstandard abbreviations used: $\alpha$-GalCer, $\alpha$ galactosylceramide; OX40L, OX40 ligand; OX40L-DC, OX40L gene-transduced DC.

Conflict of interest: The author has declared that no conflict of interest exists.

Citation for this article: J. Clin. Invest. 117:3169-3172 (2007). doi:10.1172/JCI33976. the tumor antigen itself via the TCR signaling pathway, but also costimulatory molecules (1). In the early stage of T cell stimulation, the costimulatory molecule CD28 is required for $\mathrm{T}$ cell proliferation and survival through recognition of B7 molecules expressed by DCs. "Second-wave" costimulatory molecules, represented by TNF receptor family members, are required by activated $\mathrm{T}$ cells for completion of their differentiation programs and exertion of their antitumor functions such as the release of cytokines and cytotoxic granules. The TNF receptor family of costimulatory molecules continues to be of interest in an effort to identify candidate targets for the development of immunotherapeutics. Activation of many of these signaling pathways has caused tumor rejection in preclinical and clinical studies (2).

\section{OX40 and OX40 ligand in antitumor immunity}

The OX40 costimulatory molecule is a member of the TNF receptor family and is expressed on activated CD4 ${ }^{+} \mathrm{T}$ cells and $\mathrm{CD}^{+} \mathrm{T}$ cells (1). OX40 ligand (OX40L) belongs to the TNF superfamily and is 